\title{
Communication and trust: rethinking the way construction industry professionals and software vendors utilise computer communication mediums
}

\author{
Stephen Oliver(D)
}

\begin{abstract}
Contemporary communication mediums in isolation have reached a technological plateau, proximal communication is no longer limited by spatial or technological constraints. However, integration of communication mediums and their application in pedagogy do not appear to receive the attention they deserve outwith marketing and social psychology research. This is to the determinant of AEC industries where the forefront of proximal communication extends to video conferencing and high latency sharing of open standard models. Underutilisation is not just a missed opportunity; it is the source of many cascading conflicts, trust issues and delivery failures in in AEC projects. Although effective communication will not resolve all problems, those associated with its absence are unnecessarily endemic. Through discussion of the antecedents of trust and the social presence principles that underpin effective communication, this paper proposes the next disruptive innovation in industry should be a paradigm shift in its approach to communication.
\end{abstract}

Keywords: Computer-mediated-communication, Trust, Conflict, Social presence

\section{Introduction}

Communication is the foundation of survival and essential for the growth and evolution of societies, without the ability to transmit observations or ideas animals would at best exist perpetually on the edge of extinction. Conversely, given that receiving information is an unavoidable symptom of stimuli it may be more appropriate to say the ability to interpret information is the foundation of existence. However, ultimately without both civilisations could not be formed. Communicative reciprocity whether mediated or non-mediated is the cornerstone of humanity's progression from hunter gatherers to what it would consider civilised society with each new model increasing our capacity to disseminate information, exponentially progressing civilisation both technologically and philosophically. Since earliest cave paintings to online virtual communities civilisation has developed more efficient formats improving one or more

Correspondence: stephen.oliver@strath.ac.uk

Arbnco, Strathclyde University, 75 Montrose Street, Glasgow G1 1XJ, Scotland of the core components of communication, accessibility, persistence, storage capacity, richness and latency increasing our capacity to share ideas and collaboratively innovate. Arguably, each medium has its own merits whether functional, social or cultural even in modern society and creation of newer formats will rarely make an existing medium entirely redundant, but our ability to create and integrate mediums is what defines us as a species.

Computer-mediated communication brought forth a momentous change in our ability to communicate, advancing mediated communication more in the last hundred years than civilisation achieved cumulatively. Excluding physically responsive presence in immersive virtual environments it appears its technological components, auditory, visual, textual and dissemination in isolation are primed for purpose leaving usefulness and richness as matters of integration and utilisation. As we strive for richer mediums leading towards ortho-social platforms it seems civilisation knows the end goal and has already made considerable progress in medium 
integration. However, the value of communication mediums is not in their existence and loose application alone but rather, how they are integrated into existing industries to improve their efficacy.

necessitating interdisciplinary collaborations which are not without risk. It is understandable why industry members would be reluctant to pursue advancements in their field given the risk, everyone desires to be a pioneer in their field, but in commercial settings no one wants the burden of cost in developing an innovation analogous to Betamax or leaded petrol. The construction industry has made great progress in integrating computer-mediated communication into its processes however, given its diversity in functions varying in communication model requirements, hasn't fully utilised the potential of contemporary CMC.

Nonetheless, many issues in interorganisational communication in construction projects can be attributed to failures in communication which this paper aims to show is not something that will be resolved by producing new, cutting-edge technology. Instead it will be the application and refinement of existing technologies. This suggests mediated communication will be the source of the next major industry software innovation and the disruption will likely start with centralised, concurrent access data management.

\section{Methodology}

A literature review of peer-reviewed publications encompassing trust and communication in AEC, sociology, psychology and social presence in virtual environments carried out to build a case for why the industry should focus on communication rather than cutting-edge functionality and to identify the underlying concepts behind effective mediated platform design.

Keyword identification was blind beyond the top-level concepts. Any area potentially of interest was stacked. Where relevance to previous sections was identified those sections were refined.

The paper begins reviewing the impact of conflicts in construction industries and the role communication plays. Initially investigating its overall impact and identifying the technical and social identity acting as catalysts for conflict escalation. The proceeding section considers current consensus on communication relevance in construction at a lower level from the perspective of single organisations and individuals. FinallyA review of industry-specific section on the concept of trustLatency and communication effectiveness as partially dependent properties. Discussion focuses on the hypothesis that deterioration of communication or trust can lead to a self-destructive relationship between participating and non-participating stakeholders
In the next sections the principles of media and social richness are discussed initially in terms of application before focusing on their defining characteristics. Media richness as a metric of medium efficacy is critically assessed before a progression to social presence in communication and how it translates to mediated communication. A brief discussion on the psychology of abstracting and solidifying perceptions and impact of exposure follows to suggest the notion that relationship deterioration is compounded and becomes increasingly more difficult to restore. Finally, the application of these concepts in virtual environments and human-computer relationships is explained.

The paper concludes with a discussion on the findings from the literature considering how the review sections interact and how they can be resolved. Ultimately suggesting in addition to the hypothesis that in contrast to the theme of the article, system design debatably benefits from acknowledging the social presence in mediated communication yet not truly considering users as people in the standard sense. Instead as malleable entities whose perceptions are more important than their experiences who subconsciously prefer to be coddled to an extent which is critical to communicating important information.

\section{Aims and objectives}

This paper aims to demonstrate the need for a disruptive change to the way AEC industries communication between themselves and the laity. Primary objectives are:

- Demonstrate communication and trust are heavily dependent on immediacy and proximity.

- Explain why AEC industries are suffering by not utilising contemporary computer-mediatedcommunication mediums.

- Determine whether the relationship between immediacy and the perception of social proximity favours standardised and centralised data management.

- Highlight why platforms should accommodate high richness and allow users and developers to retrieve information at a level of richness appropriate for their needs.

\section{Conflicts and relationships}

Building design and construction projects are complex and multifaceted requiring continuous feedback between teams and stakeholders which suffer the symptoms of the project issue management challenges faced by most projects (Mossalam, 2017), particularly those reliant on axiomatic design independence. AEC projects are subject to high uncertainty often resulting in destructive conflicts which have been identified as a significant 
factor in project overrun (Elziny, Mohamadien, Ibrahim, \& Abdel Fattah, 2016; Niazi \& Painting, 2017). These may incur other considerable direct and indirect costs (Lu, Zhang, \& Pan, 2015) where communication based disputes alone can contribute $3 \%-5 \%$ of total project investment expenditure (Wu, Liu, Zhao, \& Zuo, 2017). Whilst often attributable to interpersonal issues explained by social identity and uncertainty (Jaffar, Tharim, \& Shuib, 2011; Van Os, Van Berkel, De Gilder, Van Dyck, \& Groenewegen, 2015) problems are exacerbated by the typical request for information latency of 10 days, identified by Ding in 2012, cited by (Du, Zou, Shi, \& Zhao, 2018). Budget and schedule constraints inherently favour dispute settlement rather than resolution given the established cost of delays and investment expenditure. However, settlement has no real winner and although in the short term has the tangible benefit of moving the project forward it can affect inter and intra team performance (Tabassi, Bryde, Abdullah, \& Argyropoulou, 2017). They can also diminish or negate value creation (Mele, 2011), reduce participant and client satisfaction (Cheung, Wong, Yiu, \& Pang, 2011), and can lead towards conflicts which span beyond the current project. Conflicts in themselves aren't necessarily negative, functional conflicts can spur creativity (Mele, 2011; Tabassi et al., 2017; Vaaland, 2004), but they are susceptible to escalation when ill-managed. Ultimately their productiveness relies on stakeholder perceptions of one another which is largely dependent on trust and effective, timely communication (Jaffar et al., 2011; Rauzana, 2016).

\section{The impact of communication on collaborative projects and conflicts}

Effective communication management is crucial for the success of construction projects, a critical skill for design professionals (Norouzi, Shabak, Embi, \& Khan, 2015) and cornerstone function of project management (Sarhadi, 2016; Wu et al., 2017; Zulch, 2014). Conversely, ineffective communication has been identified as a source of conflict in construction projects (Jaffar et al., 2011; Mitkus \& Mitkus, 2014) including communicative ineffectiveness in the form of poor documentation and lack of trackability (Lester, 2017). In their respective, cross-disciplinary qualitative assessments of the causes behind construction project delay (Odeh \& Battaineh, 2002) and (Assaf \& Al-Hejji, 2006) found that although perceptions of primary sources of delays vary by practitioners' roles in the construction industry, communication was unanimously considered to play a critical role in overrun.

Norouzi et al. (2015) categorise communication issues between clients and architects in two groups, social and technical. They suggest the solution to social issues is the development of a framework in which data centralisation may facilitate and the development of techniques that will enable stakeholder involvement in the design process. This should encourage stakeholder participation through interactive communication activities and creating supportive features. They assert successful relationships between client and architects is often based on social relations and accessibility of information. In contrast, (Verčič, Tench, \& Tkalac Verčič, 2017) in broader terms relating to agencies and public relations departments found that clients were more concerned about the agency's understanding of their business, performance and frequency of errors. This was surprisingly near polar opposite of the participating agencies' understanding, and clients were least concerned about interpersonal relations. Although not entirely homogeneous and may be presumptuous of a swift trust relationship between clients and construction contractors these may offer to important insights into general client-contractor agreements. Clients are businesses and at the end of the day which don't need to make friends to achieve their goals. Their business is their primary concern and interpersonal relationship components are perhaps not as significant as expected by both Norouzi et al. or this article.

Bourne (2016) suggests effective communication requires audience targeting, identifying what defines the audience on an individual level and attempting to best accommodate their communication expectations. Her work expands on normal discussions on essentially social presence concepts but puts significant weight on the subjective nature of people's perceptions and unique preferences for communications models. Thus suggesting that effective communication necessitates assessment of the individual given communicative interactions are subjectively experienced and cannot be tailored based on the consensus. The article appears relevant to this discussion and one loosely defined consideration arises that in contrast to the impetus of this article, it may be possible to provide stakeholders too much information.

\section{Trust in interorganisational settings}

*Trust in organisational relationships is multidimensional in both attributes and network models that extends beyond what would be reasonable discussion for this paper. The scope of this section is limited to generalisations from the literature unless the subject necessitates a distinction.

Trust is a fundamental aspect of communication, negotiations, appraisal of performance, labour relations, project management and client/contractor relations; it is an effective mechanism enabling teams to work efficiently especially for diverse, and distant and/or distal teams or collaborations. (Cheung et al., 2011; Jarvenpaa, 
Knoll, \& Leidner, 1998; Mayer, Davis, \& Schoorman, 1995). Expanding on earlier definitions of interorganisational trust, Mayer et al. suggesting defining it as the willingness of a party to become vulnerable through delegation of an action to a third party regardless of the trustor's ability to monitor or control the trustee's actions later making the necessary distinction between cooperative and trusted actions. The former denoting an act that doesn't necessarily require trust or has no perceived risk. Similarly, they make distinctions between trust and predictability where the latter is the trustee's conformity to the expectations of trustor Finally, they introduce the concept of confidence where confidence is a lack of consideration for the outcome, arguably a high-level construal formed by consistent predictability of beneficial outcomes for the trustor. Although this definition appears universally applicable, in contracting it is often defined as the willingness to share information (Cheung et al., 2011).

Generally, trust has been shown to improve performance (Weinhofer, 2007) and perhaps more pertinently can in the context of competence trust, result in a higher probability of projects being completed on schedule with performance satisfaction complementing contractual safeguards (Steven \& Hang-Yue, 2004). However, the relationship appears to be inverse for goodwill trust. The key aspects of trust in terms of this article are that trustful organisational relationships are less distal, create collaborative, problem-solving and cooperative environments, and greater information sharing between parties (Weinhofer, 2007). These characteristics appear complementary to the concepts of media richness implicitly enrichening mediums in equivocal tasks regardless of medium given that richness is characteristically attributable to presence (Bergin, n.d.) which is partly a function of intimacy and immediacy (Weiner \& Mehrabian, 1968).

(Jarvenpaa et al., 1998)'s study on the antecedents of trust in global virtual teams identified many of the characteristics assumed to be congruent to communication at the conception of this article. They found the high-trust teams were proactive in their actions, shared responsibility, made better use of time, provided consistent substantive feedback, focused on output and flexible. At the core of all three high-trust groups was substantive and frequent communication. Though these groups did have conflicts they were resolved with minimal disruption which can seemingly be attributed to the communication model as much as the trust itself, whereby they openly discussed interpersonal concerns as they arose. Their observations appear to concur with common perceptions of the characteristics that define successful collaboration in the construction industry (Mesa, Molenaar, \& Alarcón, 2016; Suprapto, Bakker, Mooi, \& Moree, 2015). In their discussion, Jarvenpaa et al. cite
Mayer et al. (1995)'s argument that trust is directly impacted by the antecedents of trust, interpreting it to suggest that inactivity may interpreted as a lack of trust or reliability. One final observation was worth noting is that as the projects progressed the salience of team members' ability became less significant, this may support a suggestion from this article that social exposure and creation of construals (from "They attempted this, achieved..., They attempted this, achieved......." to "they are capable of") highlight the importance of immediacy, but the scope of their study is not sufficient to generalise the concept suggested in this article.

\section{Media richness in computer mediated communication}

Computer mediated communications (CMC) were received with mixed reviews as they gained traction in the '70s. Concerns were raised over the impersonal nature of emails, loss of workplace agency and general privacy to the extent that the Swedish Data Inspection Board set a time limit on the duration people were permitted to store emails on their computer (Palme, 2011). According to Media Richness Theory (MRT), the theory that equivocal and unequivocal communication are best handled by rich and lean media formats respectively, CMC is a varyingly effective form of communication. On the surface it considers email a particularly ineffective medium for communication based on its five criteria of effectiveness [medium, feedback, channel, source and language] (Bergin, n.d.; Daft \& Lengel, 1983) and synchronous video CMC (SVCMC) to be closer to the effectiveness of face-to-face (FtF) communication, an observation supported by (Suh, 1999). However, when considering individual features of media formats it is apparent that the general MRT definition of effectiveness ignorant of satisfaction doesn't adequately value text communication and media cannot be evaluated exclusively in terms of richness (Kishi, 2008; Otondo, Van Scotter, Allen, \& Palvia, 2008) without consideration for task and purpose.

Further compounding debate over the applicability of MRT (Palvia, Pinjani, Cannoy, \& Jacks, 2011) find contextual constraints play a significant role in media format preference, noting a salient point to this article of text-based CMC's important role in accountability. Saat and Selamat (2014) go as far to say attempting to generalise media richness is meaningless. Dennis and Kinner (1998) agree with the consensus but highlight a relevant observation for this article, high equivocality tasks benefit from high richness media formats and low-latency communication.

In contrast, (Saat \& Selamat, 2014) found MRT supported assessment of media richness relevance in presenting corporate information to public audiences seemingly supporting the social presence aspect of MRT. 
Similarly, MRT was shown to be consistent in interactive teaching of equivocal subjects (Chang, Liang, Chou, \& Lin, 2017; Liu, Liao, \& Pratt, 2009; Sun \& Cheng, 2007). Two final noteworthy observations come from (Joseph \& Janet, 1991) who identify perception of richness as metric for predictive validation, supported by (Anandarajan, Zaman, Dai, \& Arnzie, 2010) contradicting the underlying assumption that richness is an objective characteristic, and social influence impacts the uptake and effectiveness of CMC. The latter may to some extent explain the lower than expected uptake of rich media formats in the construction industry.

Perception was found to be particularly interesting in one generally supporting article that found not only does richness have a positive impact on those receiving the information but interactivity was not a significant factor in determining effectiveness which they suggest may be a symptom of perceived interactivity (Lu, Kim, Dou, \& Kumar, 2014). MRT, at least in the context of organisational communication is too simplistic for assessing the effectiveness or value of media formats in general terms. However, the literature provides valuable information on the nature of organisational communication highlighting that selection of appropriate media formats is crucial for performance, requires situational and contextual considerations, and counterintuitively does not consistently correlate with the aspects Social Presence Theory (SPT) to the extent asserted in earlier research.

\section{Social presence and mediated communication}

Social presence is defined by (Short, Williams, \& Christie, 1976), cited by (Lowenthal, 2009) as "the degree of salience (i.e., quality or state of being there) between two communicators using a communication medium". Gunawardena and Zittle (1997) offer a refined definition "the degree of which a person is perceived as a 'real person' in mediated communication" which appears to be the framing definition used in later literature including Lowenthal's. Short et al. identify, citing (Argyle \& Dean, 1965) and (Weiner \& Mehrabian, 1968) for each component respectively, intimacy and immediacy as the two primary components of social presence. (Rafaeli, 1988) later formalised the notion of interactivity as a third component representing the reciprocal aspect of FtF communication, occasionally described as ortho-social in the literature. Social presence, more commonly than media richness is found to be effective in task performance through the mere presence of others. However, it is nonetheless situational and may impede performance (Garcia-Marques, Fernandes, Fonseca, \& Prada, 2015). Social presence in media communication is an inherently important consideration for communication models which can have online-invisible, knock-on effects which manifest in non-mediated interactions (Sutcliffe, Binder, \& Dunbar, 2018).

Immediacy is the subjectively experienced (Trope \& Liberman, 2010) measure of psychological distance (distality) between the communicator and the object of their communication measured through four psychological distances, spatial distance, temporal distance, social distance and hypotheticality (Liberman, Trope, \& Stephan, 2007) which have been observed to be adaptive to the individual's perception of physical and social contexts (Chen, Mitchell, Brigham, Howell, \& Steinbauer, 2018). Perception of distality is automatically assessed and utilised regardless of relevance to the task at hand (Liberman \& Trope, 2014). Gunawardena (1995) in her study of social presence as a predictor of teaching quality concludes there is a positive relation between immediacy and affective and perceived cognitive learning supporting previous discussion on MRT's value as a metric of media format efficacy in educational settings.

Intimacy has various forms differing for adults and children in both somatic and social, and physical and psychological categories. Adults looks for intimacy through shared experience and perceived social similarity, to reciprocally share experiences. Conversely, children seek individuality or a sense of self. It is divisible additionally into somatic and non-somatic perceptions dealing with the connection of body and mind and social connection with others. In the context of social presence in professional use of $\mathrm{CMC}$ it is worth focusing on the psychological with physical aspect technologies predominantly focusing on mediated interaction rather than interpersonal communication, though somatic intimacy inherently plays a large role in telepresence. Intimacy is generally considered closeness and familiarity involving close relations, but interestingly has also been described as the willingness to accept that the mind of another is uninhabitable to the self. The latter may show the value of non-somatic characteristics as the key precursors for presence where email, telephone and video conference improved sequentially seemingly as hypotheticality is reduced through the introduction of additional sensory interpretation of individual verbal and nonverbal components of communication (Gunawardena \& Zittle, 1997).

Interactivity is the capacity of an entity to facilitate active participation for present parties, essentially unrestricted, asynchronous and disordered access to stimuli. Another way to think of may be the introduction of low-latency uncertainty. It coaxes engagement from participants mixing methods for letting the user set the level of guidance through information sets and feel like they are in control. In principle it introduces variable human-environment information bandwidth enabling participant expeditions through the information at their 
own pace. It can dictate user attention, ability to recall information and exposure time however, its impact is non-linear (Xu \& Sundar, 2016). From the media richness discussion by (Joseph \& Janet, 1991) it was shown to be widely open to interpretation with perceived interactivity, a psychological construct of perceived self and external system efficacy (Vendemia, 2017) to have notable significance. In professional settings interactivity may share similarities with the voice effect (Roberts, Lowry, \& Sweeney, 2006), people want to contribute or "be heard" even if it has not impact.

\section{Abstraction and solidification of perceived distality}

Construal Level Theory attributes distality to the formation of mental abstractions (construals), the evolution of memories changing to and from subordinate and superordinate observations in correlation with distality (Trope \& Liberman, 2010). Trope and Liberman offer as an example, 'a proximal observation of "they waved at me" may become a distal "they displayed friendliness". This seems particularly relevant to discussion on immediacy in disputes or discussions since abstraction whilst capable of adding additional context to the observation, can result in the omission of inconsistent details (Liberman et al., 2007) and higher level construals are less likely to change as distality increases or decreases (Trope \& Liberman, 2010). Reyt, Wiesenfeld, and Trope (2016) explain the difference between levels as a contextualised "how" and decontextualized "why" for lower and higher levels respectively. In the context of collaborative disputes as low-level "we will meet to discuss the options" and "overruns are costly, and a resolution was necessary to continue work". Reyte et al. highlight the possibility of using the Linguistic Category Model (LCM), a set of rules to calculate the level of abstraction in text as a means of inferring a person's construal level based on the language they have used discussing a given subject which may be a generally useful skill.

\section{Presence as a virtual environment concept}

Discussing social presence in the context of virtual environments (Biocca, 1997) suggests perception of might be summarised "The minimum level of social presence occurs when the users feel that a form, behaviour or sensory experience indicates the presence of another intelligence. The amount of social presence is the degree to which the user feels access to the intelligence, intentions and sensory impression of another". Biocca highlights the usefulness of this description citing (Husserl, 1973) as a relation to the human capacity for empathic perception of another through nonverbal cues, indicating that the measure should be the level in which communication between the person and another feels like FtF. For high level discussion on presence, Biocca notes two additional forms of presence, telepresence, the experience of being at being there in the now and co-presence, the feeling of being in the company of others at a fundamental level not necessarily encompassing all aspects of social presence, citing (Schroeder, 2002). Schroeder discusses telepresence using the general terms "presence" and "being there" without explicitly using "telepresence". Schroeder explains these concepts are intrinsically linked forming social presence, a term he suggests to be more relevant for non-immersive environments, in contrast to (Nowak \& Biocca, 2003) who maintain a distinction between the three forms of presence. (Lombard \& Ditton, 1997) define six overlapping explications of immersive presence:

\section{Social richness}

A concept which is effectively a for-purpose adaption of SPT assigning greater importance to the number of senses involved and the interface's capacity to immediately provide feedback. The distinct prominent feature of this concept appears to be co-presence, the sense of being in the company of others through mediated communication described originally by Goffman 1963 "copresence renders persons uniquely accessible, available and subject to one another" (Nowak \& Biocca, 2003) as a purely psychological connection between people.

\section{Realism}

The measure of being perceived as if a real-world experience. This encompasses the perceptibility of the surroundings and stimulation of audio-visual senses. They identify two sub types of realism, social and perceptual. The former is the sense of feeling real in the sense that the events could believably occur in a non-mediated environment. The latter being perception of the events with social expectations suspended, ignoring if the event could happen but rather did the physical sense stimuli elicit a feeling that the event could be real. Although not mentioned by Lombard and Ditton, diegetic audio-visual and binaural audio are likely significant factors for both social and perceptual realism. In a study on menu interfaces for virtual reality (Santos Torres, Zarraonandia, Díaz, \& Aedo, 2017) acknowledge diegetic menus as a mechanism for increasing the sense of presence identified in previous literature however, they question whether they are efficient but unfortunately they do not include this in their research. (Ballestero, Robinson, \& Dance, 2017) found head-tracked binaural audio can lead to greater immersion in virtual environments. However, realism does not appear to always have a positive contribution to the perception of presence. In contrast to previous research by CyberArts and discussed by (Heeter, 1992), (Nowak \& Biocca, 2003) found that increasing anthropomorphic realism in avatar design may lessen the experience by raising expectations, noting the participants in the study found avatars less human in appearance to contribute to a better sense of presence. However, CyberTech (Heeter, 
1992) found that in second person VR participants preferred real representations of themselves. (Cliffard, Steuer, \& Tauber, 1994), whilst researching ethopeia in relations to computers suggest concerns about the necessity for higher levels of realism may be too conservative. Similarly, (Lee \& Nass, 2003) note people are fairly liberal when it comes to assigning humanity to machines further suggesting that realism may be lower in the order of synthesising social presence in VEs.

\section{Transportation}

Telepresence, most commonly referred to as "being there" yet described as "you are there" in their research. From the literature they identified a common description of "a form of out-of-the-body experience". They note from Kim T 1996 (an unpublished doctoral thesis from University of California) that the common descriptions from participants suggesting a metric were the "departure" from non-mediated and arrival to mediated environments. Their discussion whilst mainly focusing on televised media also found a counter interpretation "it is here", where the participant felt that the world came to them rather than being transported to the world. The term, according to (Steuer, 1992), appears in earlier literature to describe remote interaction with physical objects seemingly diverging in definition in 1992 when (Heeter, 1992) introduced the term as a virtual environment concept. (Biocca, 1997) discusses response systems as a mechanism for creating a physical sense of presence. Sensors track the user and mirror their actions in the virtual world.

\section{Immersion}

Immersion is the psychological and perceptual sense of being removed from the real world and existing in the mediated world. Essentially, the measure of sensory saturation from the mediated environment or desaturation from real world stimuli (Biocca, 1997). They cite (Loomis, Blascovich, \& Beall, 1999)'s description of perceptual immersion as "The degree to which a virtual environment submerges the perceptual system of the user". Loomis et al. observe the potential for immersive virtual environments (IVEs) as a mechanism for reducing trade-offs between experimental control, they appear to indicate perceptual immersion is a primarily a function of the utilisation technological capacity and capability of the development team behind the experience. Psychological perception in contrast is the more subjective perception of involvement, engagement and engrossment. They cite (Heeter, 1995) who identified in her study that people weigh these characteristics in terms of fun, competition, excitement, addictiveness and intensity. Heeter also identifies common negative characteristics of social discomfort in the form of embarrassment, silliness and intimidation, and overload from feeling confusion and overwhelmed.

\section{Para-social interaction and relationship development}

A concept attributed to Horton and Wahl's (no reference in Lombard \& Dittion's paper) work in 1956 on SPT where they coined the term "para-social interactions" (PSI), covered by (Klimmt, Hartmann, \& Schramm, 2006). People develop one-sided, non-dialectic relationships (PSR) with characters from media discussed by Klimmet et al. in the context of tv personalities (PSI), computer game (PSR) characters and software-embedded instructors (PSR), considered to be complementary to social relationship development. In his review of the literature (Giles, 2002) found two contributory functions associated with the PSI which seem moderately relevant to PSR, companionship and personal identity. Broadly, the phenomena stems from aspects of SPT and Social Cognitive Theory (SCT) discussed in the context of more conventional media formats in Entertainment Theory (Klimmt et al., 2006) that is developed through similar mechanisms to presence as currently being discussed. For effective PSR/ PSI characters are developed to evoke empathic links with the persona attempting to brew investment in the character non-dialectically from the consumer where the consumer engages in anticipatory observations and judgment resulting in a relationship between the persona and the self (Klimmt et al., 2006; Rubin \& McHugh, 1987). It is worth considering if this is more important than other forms of perception at least in the context of VEs with medium to long term involvement where investment in the persona/instructor/character is pertinent to media effectiveness. This may be an interesting opportunity for further research into the significance of this form of perception in comparison to realism in IVEs given (Nowak \& Biocca, 2003)'s observation on anthropomorphic realism in avatars. PSRs can have compounding impacts on the perception of social presence in online gaming communities. Basing their study on the Social Identity Theory (SIT) premise that people have a strong, hard-wired motivation to identify with groups and the tendency to prefer people meeting certain templates found people develop a stronger sense of social presence when they identify with their avatar whether drawing similarities between themselves and the avatar (high self-esteem) or seeing qualities they want to see in themselves (low self-esteem).

\section{Medium as a social actor}

The final and likely most nuanced form is the medium being perceived as an actor or persona seemingly exhibiting social presence, alternatively referred to as the computer as social actors (CASA) paradigm. On the surface this appears to be anthropomorphism however, this was rejected in favour of ethopeia on the determination of human interactions with computer being subconscious rather than mindful (Wang, 2017). The distinction arose from the observation that whilst humans 
apply social rule and etiquette subconsciously when interacting with computers, they are ever-aware of the fact that it is not a living entity they are communicating with and it does not necessitate humane treatment (Nass \& Moon, 2000). The perception of social presence from machines is surprisingly intense even in cases where the social cues accommodated by the machine were limited with (Cliffard et al., 1994) observing the application of gender stereotypes and (Nass \& Moon, 2000) finding supporting evidence going further observing the application of ethnic stereotypes. CASA is possibly the most complex of the six presence explicates. Noting the liberal personification of computers (Lee \& Nass, 2003) identify the granularity of developing voice-mediation in effective embodiment of human traits. (Wang, 2017) in a reasonably positive study allude to three disheartening observations. 1) Personification may lead to over-attention to a machine at detriment to real-world relationships. 2) People can be quick to dehumanise devices as soon as they behave undesirably from a technological standpoint. 3) The universal issue problem that people will always find ways to draw controversy regardless of scientific basis or intent; they note Apple's choice to give Siri a female voice in several countries, appropriate based on SIT, SCT, and CASA research, was received with criticism. It is likely one of the most interesting design considerations for computer scientists that social ideology can be paradoxical.

\section{Discussion}

Improvements to communication in AEC models are discussed frequently in the literature highlighting a known need for better communication between stakeholders. Higher social presence in software design does not guarantee project success but it does provide a mechanism for solving the problems that are commonly discussed and facilitating the solutions suggested. Norouzi et al. (2015) offer the following common failures that breed mistrust and conflict between designers and clients which are proceeded by suggestions as to why better communication through social presence in software may mitigate these issues.

\section{The client's viewpoint is not understood}

Facilitating interactivity and low-latency communication between stakeholder ideas enables thrashing out ideas and letting each party to discuss interpretations in real-time or otherwise reasonably short intervals. Furthermore, accessibility to information outwith direct communication between designers and clients will allow clients to explore designs and concepts in their own time. Though not discussed in previous sections interactivity could be very efficient in breaching the industry knowledge barriers through tooltip integration with laity-friendly information on the concepts that lead to the decisions made by designers. Solar shading may exemplify the value in these features. By providing the client with short tooltips and toggleable shading positions they can understand and see for themselves the impact of various solar control measures.

\section{There was not enough communication between stakeholders}

The running theme of this paper is solutions for resolving this problem. Visualisation increases client-contractor information bandwidth reducing the amount of time required to convey and discuss concepts and ideas. Interactivity allows clients to show the designer where there is conflict between their interpretations of ideas or the designer to demonstrate technical constraints in a manner which may be easier to understand. Though it is currently unclear to the author how effective software developer can be in designing interactivity that doesn't require foreknowledge of the environment to the extent that the client would become disinterested, perhaps at this stage aided interactivity during bidirectional communication would be most fruitful.

\section{Design requirements were not sufficiently managed}

This subject requires little discussion. It is clear from the evolution of software and the introduction of standardisation and interoperability that effective management requires improved information management and presentation which is an inherent feature of social presence considerations in software design.

\section{The needs expressed by the clients often change}

Reducing latency in client-contractor communication will to an extent mitigate the impact of client requirement changes by allowing the client to ensure their change requests are understood and delivered in a timely manner. This may also be improved through interactivity and asynchronous access to the information by the client, who may be able to rehearse or otherwise investigate in the virtual environment prior to attempting to convey the information to the designer.

\section{There is a lack of feedback from the client}

This is a complicated problem which ties back to all aspects of the literature reviewed in this article and the solution discussed regarding the previous complaints. Generally, though, it could be argued that it is a lack of effective communication tools that can allow the client meaningfully to understand the technical aspects of the project and produce communications which are valuable to both parties, most importantly in a cheap and non-obstructive manner. Current processes requiring both parties to be present don't effectively facilitate such 
activity. There is a certain l'esprit de l'escalier (escalator thought) type feature of current meeting-based communication whereupon the client may find the answer or retort they were looking for only after the meeting has finished. In their paper, Norouzi et al. note Shen's (2011) proposed solutions which run in theme with this article.

However, the author acknowledges that this relies on the assumption of either existing trust or confidence and is susceptible to the same social identity issues known to be at the root of some conflicts. This is evident where established relationships are not present such as the introduction of a newcomer to a project. Whilst eagerness has been known to be well-received it has likewise been received with suspicion and resistance (Van Os et al., 2015) which may be exacerbated by the low-latency, asynchronous data access suggested in this article since it does not ensure reduced temporal or social distality and thus can increase hypotheticality. On one hand, asynchronous, low-latency access prevents the unwillingness to share information resulting from conflicts which may exacerbate failing trust, on the other it shifts the professional nature of relationships and may lead to cascading conflicts where more traditional, ostensibly subversive information and expectation management would not. Effective communication is the cornerstone of preventing cascading conflicts but it is evident from (Wu et al., 2017) that this article may be too optimistic of technology's ability to alleviate social identity and information confusion related escalation.

\section{Permit the client to feel as though their contributions are valued and involved [the] stakeholder}

This is the voice effect in action. People don't necessarily want or need their contributions to be the overruling opinion on a given subject however, it is important to people to feel as though they have been involved in the process. Better communication software features as discussed in this article both facilitate greater collaboration and reduce the social distality between parties which may otherwise lead to a feeling of being less valued by the opposite party. Part of this is likely achieved through the previously discussed benefits of interactivity and asynchronous access whereupon the client may investigate design features or constraints that may feel overwhelming during face-to-face interactions when the concept being discussed is new territory to them.

\section{Effectively administer changing design requirements}

This is largely a feature of low-latency in information dissemination though arguably a side effect of previous considerations. By making changes available to all parties as soon as they have been implemented would permit clients to review changes in a familiar environment quickly to ensure there is no discrepancy in interpretation of the requirements.

\section{Employ appropriate and easy to understand visualization techniques}

This proposed solution embodies many of the running themes of this article. The keyword from the statement is appropriate. It appears to offer a case for the top-down software design approach lightly touched upon in this article. By creating models which can be interacted with by many design tools the features of low-latency, asynchronous communication can effectively be leveraged in the appropriate context whilst still serving the general purpose of enabling effective communication.

It is clear from the literature including Norouzi et al. that though there are both social and technical barriers in client-contractor communication, it is largely technical barriers that are the primary concern given previous observations on the client's seemingly low interest in social distality. However, social distality eases the tension between parties, creates a better collaborative environment and should improve the voice effect which is arguably one of the more significant features of effective communication. Norouzi et al. conclude that the running themes of this article is pertinent to both client-contractor relationships and effective architectural design.

The literature recognises the need for communication study of AEC interactions and that communication is a critical factor in all aspects of construction projects (Norouzi et al., 2015) whether the teams involved are close-knit or global virtual seemingly related to its effect on proximity or in general terms, social immediacy. Destructive conflicts are largely attributed to communication failures which alone can result in significant overrun and increased costs. In contrast effective communication is the fundamental component of functional conflicts which can result value creation, creativity and net gain for all parties. The main contributory factors of project performance are interlinked with communication at all levels of the project hierarchy ranging from the individual to the collective organisational network. One of the key properties of communication was low-latency, substantive interaction between teams (Dennis \& Kinner, 1998) which fostered the development of positive relational attitudes, trust, independence, understanding and inherently reduced the potential negative effects of hypotheticality on relationships. The observations are supported by the sociology, psychology, communications and construction literature, industry participants in project management research and clients. Though the social relationship aspect was inversely valued by client and contractors which is surprising given impact on social presence and trust. It would seem clients at least in self-reporting that social distality for them is not as 
much of a social identity consideration as was initially believed. One paper in the literature suggested that communication is more granular than both this article and as commonly presented in the literature that requires a personal-level, targeted communication model. Whilst this initially seems self-evident considering the difference in perceptions between clients and contractors on interpersonal social relationships, it appears that generalisations are made about perceived client needs rather than what a client wants. Finally, the literature identifies the necessity for a social communication framework for client-contractor interactions indicating the value.

Trust and effective communication go hand in hand, although neither guarantee improvements in the other it does appear that they are interdependent properties. Communication's impact on trust may be attributed to the how substantive the information is and how well the aggregate of the message is presented over periods in a communication chain. It can define perceptions of ability, integrity and benevolent trusts though whilst benevolence can be an indicator of dyadic trust it may be abused at interorganisational level. Trust is similarly related to relational attitudes which was identified to enable functional conflicts, creativity, value creation and most importantly makes teams less distal which bridges cultural and social gaps between groups. Although it cannot be said with certainty and is merely a postulate in this article, trust may have an inherent impact on the perception of media richness given that richness is attributable to social presence and the observations of trust and communicative effectiveness appear to reduce the distality factor of presence. If this is the case then not only does improving these features of a construction project improvement performance and satisfaction (Gunawardena \& Zittle, 1997), they should improve the perception of richness in CMCs of the involved parties which may, in cases of equivocal tasks, improve the learning aspect of project communication. This should also contribute to the perception of trust and communicative effectiveness. However, it should be noted that media richness was shown not to be a reliable metric of efficiency in CMC outwith education contexts, though the literature did not appear to identify instances of a negative relation between richness and efficiency beyond the implicit link between the observation on too much interorganisational benevolence and opportunity for relationship abuse. Most of these concepts are covered partially in isolation but have not been viewed collectively and a broader psychological aspect is absent. Peoples' opinions and interpretations of others and teams are subject to social exposure and the development of construals as they become distal. It was not the intent of this article to investigate this however, both social perceptions and mental abstractions are difficult to change once they are formed suggesting that the discussed aspects of relationships linked to communication should be taken into from the offset and remain consistent, especially where conflict arises. However, a necessary distinction must be made between the application of synchronous and low-latency, asynchronous communication in these areas, and medium-latency in decision making. Whilst the former has intrinsic benefits in effective communication of ideas and facilitation of discussion, effective decision-making benefits from medium-latency intervals in communication. This applies in terms of both the decision maker's capacity to comprehend and assess information, and the willingness of other parties to accept their response (Grimm \& Mengel, 2011).

Everything in communication beyond transmittance of unequivocal information is perceived subjectively by participants, a characteristic which does not seem to get the attention in industry software it demands (Norouzi et al., 2015). This is a problem since the experiences of engagement with software and ability to absorb information are improved as presence is refined and proximity increases, demonstrating the need for rounded and tailored environments not necessarily on the cutting-edge. Instead software primed for appropriate presence in the context of the purpose of the data exchange between environments and/or other participants. It is understandable from a software development perspective in the sense that there is a practical limit to purpose specialisation and from the construction industry which suffers reluctance to change their existing models. Essentially, it seems risk aversion even with relatively low risk impedes progress. However, centralisation of data and standardised interfacing through APIs with concurrent data structure access opens the market up to specialists not necessarily involved in construction industries should spur innovation. Centralisation is the foundation of accessibility and seemingly gives the perception of higher social proximity when users can access information asynchronously. However, centralisation whilst the implicit solution is not necessarily the only solution, specifically the solution appears to be a matter of the relevant richness and accessibility of desired information rather than centralisation of all information. The author does, however, maintain that at least from an innovation standpoint, developers will significantly benefit from centralisation akin to the benefits reaped from the internet. That is, development can be as much a mining exercise as it is targeted.

Immersion in professional communication should weighted towards audio-visual copresence and visual channel stimuli and interactivity telepresence in collaborative or equivocal discussions. This consistent with cue-summation (Xu \& Sundar, 2016) and MRT in education (Saat \& Selamat, 2014). People prefer avatar realism when communicating via video conference however, in immersive virtual environments avatar realism increased expectations reducing the sense of telepresence and realism suggesting video conference embedding is a more appropriate choice. However, effective diegetic medium embedding in IVEs is best 
handled in different ways depending on the purpose of immersion. Interactivity plays and important part in telepresence and effectively participant-optimised human-computer bandwidth management but there is a turning point where increasing interactivity negates its value (Xu \& Sundar, 2016), people want some level of guidance whilst avoiding information overload.

Virtual spatial proximity was suggested to show reduction in the perceived spatial and temporal distance which in turn enriches the perception of richness in communication mediums. Spatial and temporal proximity appear to define human-computer / client-contractor interaction bandwidth and ability to consume information whereas social distance may improve the reception of information. Hypotheticality seems to be a symptom of the others which is possibly the most relevant feature of immediacy in construction industry communication failures. Unless there is established trust or confidence and/or social proximity the party awaiting response will increasingly become more distal and detached from the reality which will have a residual effect on future communications becoming increasingly difficult to negate (Trope \& Liberman, 2010).

Standardised, top-down data management reduces the risk for the developers and accessibility of the construction industry for the laity by guaranteeing medium-term security for data handling and interoperability and removing vendor-lock. This is the primary reason the proposed shift in development focus should occur and sooner rather than later. Standardisation spurs economic growth and innovation (Blind, 2013) and accessibility (interoperability, availability and access) spread awareness, therefore potential for innovation in a self-improving loop procedure.

Accessibility is fundamental to trust and engagement. Throughout the literature it is clear that at best restricted access is inconvenient, if not short-sighted whether temporal, spatial or social expectations are not met. In contrast, accessibility was shown in both construction and learning to entice learners, improve information retention, recognition, guide information consumption, increase team efficiency and reduce the risk of escalation to dispute between parties. Part of this is just comfort, people like to feel in control of their actions however; they engage and learn more with being coddled to an extent. If a person has spent time programming they will likely have a sense of self efficacy perceiving their time with documentation and educated guessing as independence. However, upon closer inspection they were guided by partial google searches, plagiarism on stackoverflow, popular opinion, friends' experiences and so forth. This is not to discount developers' capabilities, humans learn through exposure and the ability to expose themselves to relevant information is critical in learning. The point is moderate interactivity and availability have been shown to increase learning capacity and focus users on targeted information, propagation benefits from being targeted whilst consumers benefit from perceived self-efficacy. Most significant features of social proximity in CMC exist in isolation or merged in other specialisations however, their application is still juvenile in many cases. Unlike any period in history we have the capability to implement the most significant features of high, non-tactile social presence. Therefore, it seems the most effective way to improve communication and information propagation is to focus on tailoring mediums and experiences to the audience.

This suggested approach is not necessarily a precursor for the next disruptive innovation in industry communication given developing pedagogy takes time, trial and error. Even small sets of people can be vastly contrasting leaving risk and requiring procedure refinement. But there is evidence from the literature and the historical development of mediated communication that the result will be a net gain for society. It is up to the dominant industry software vendors to decide if the initial risk outweighs the social and subsequent client private benefits.

\section{Conclusion}

This article provides a review of the literature surrounding human-computer/client-contractor interactions and socialisation to support the proposal that communication effectiveness and standardisation are the foundation of the next major disruptive innovation in AEC industries through creative application of existing technologies and data and access concurrency. It appears social, communication, industry, computer science and psychology research is partially aware of the void in innovation and their relations however, there is a lack of collaboration. Communication mediums will continue to evolve with or without the industry, but it will miss out on growth and the potential for innovation both internally and for society. People are complex entities whose optimum capacity to consume information will be determined by the social innovation of forthcoming technology. Communication has been shown to be a primary issue with project disruptions, therefore it seems reasonable to suggest that future industry software design should start to shift focus from cutting-edge functionality to social presence integration and reduced latency.

However, it must be acknowledged that the trust and conflict in AEC literature is less optimistic about stakeholder willingness and capacity to utilise communication utilities objectively and highlights how effective communication is not exclusively a technological challenge. This article focused on the technological aspects of conflict mitigation through social presence however, the literature indicates that even with complementary pedagogical advancement the article's premise cannot fully mitigate 
social identity conflicts or stakeholder politics, and may introduce volatility preventable through effective application of existing mediated communication procedures.

\section{Abbreviations}

AEC: Architecture, engineering and construction; CASA: Computers as social actors; MRT: Media richness theory; PSI: Parasocial interactions; PSR: Parasocial relationships; SCT: Social cognitive theory; SIT: Social identity theory

\section{Acknowledgements}

The author would like to recognize the support and tolerance of $\mathrm{M}$. Eisbrenner of arbnco and Strathclyde University which has enabled the author to pursue their academic and professional interests.

\section{Funding}

This research project was not funded.

\section{Availability of data and materials}

All reference materials readily available online via Science Direct and other similar services identified via Google.

\section{Authors' contributions}

The paper is the sole works of the named author, no other party was involved in the research or writing. The author read and approved the final manuscript.

\section{Competing interests}

The author declares that he has no competing interests.

\section{Publisher's Note}

Springer Nature remains neutral with regard to jurisdictional claims in published maps and institutional affiliations.

\section{Received: 15 June 2018 Accepted: 16 May 2019}

\section{Published online: 01 June 2019}

\section{References}

Anandarajan, M., Zaman, M., Dai, Q., \& Arnzie, B. (2010). Generation Y adoption of instant messaging: An examination of the impact of social usefulness and media richness on use richness. IEEE Transactions on Professional Communication, 53(2), 132-143.

Argyle, M., \& Dean, J. (1965). Eye contact and distance affiliation. Sociometry, 28(3), 289-304.

Assaf, S. A., \& Al-Hejji, S. (2006). Causes of delay in large construction projects. International Journal of Project Management, 24(4), 349-357. https://doi.org/ 10.1016/j.jproman.2005.11.010

Ballestero, E., Robinson, P. \& Dance, S. (2017). Head-tracked Auralisations for a dynamic audio experience in virtual reality sceneries. 24th International Congress on Sound Vibration. London: London South Bank University.

Bergin, R. (n.d.). Media richness theory. Center for Homeland Defense and Security. Department of National Security Affairs. https://www.chds.us/coursefiles/ IS4010/lectures/tech_media_richness_long/story_content/external_files/ Media\%20Richness\%20Theory\%20script.pdf

Biocca, F. (1997). The cyborg's dilemma: embodiment in virtual environments. In Paper presented at the proceedings second international conference on cognitive technology humanizing the information age.

Blind, K. (2013). The impact of standardization and standards on innovation. Nesta Working Paper Series, 13(15), 17-23.

Bourne, L. (2016). Targeted communication: The key to effective stakeholder engagement. Procedia - Social and Behavioral Sciences, 226, 431-438. https:// doi.org/10.1016/j.sbspro.2016.06.208

Chang, C.-C., Liang, C., Chou, P.-N., \& Lin, G.-Y. (2017). Is game-based learning better in flow experience and various types of cognitive load than nongame-based learning? Perspective from multimedia and media richness. Computers in Human Behavior, 71, 218-227. https://doi.org/10.1016/j.chb. 2017.01.031.

Chen, H. S., Mitchell, R. K., Brigham, K. H., Howell, R., \& Steinbauer, R. (2018). Perceived psychological distance, construal processes, and abstractness of entrepreneurial action. Journal of Business Venturing. https://doi.org/10.1016/j. jbusvent.2018.01.001.
Cheung, S. O., Wong, W. K., Yiu, T. W., \& Pang, H. Y. (2011). Developing a trust inventory for construction contracting. International Journal of Project Management, 29(2), 184-196. https://doi.org/10.1016/j.jproman.2010.02.007.

Cliffard, N., Steuer, J., \& Tauber, E. R. (1994). Computers are social actors. In CHI '94 proceedings of the SIGCHI conference on human factors in computing systems (pp. 72-78).

Daft, R. L., \& Lengel, R. H. (1983). Information richness. A new approach to Managerial Behavior and Organization Design. Texas A and M University College Station. College of Business Administration. Amsterdam: Elsevier.

Dennis, A. R., \& Kinner, S. T. (1998). Testing media richness: Theory in the new media: The effects of cues, feedback, and task equivocality. Information Systems Research, 9(3), 256-274.

Du, J., Zou, Z., Shi, Y., \& Zhao, D. (2018). Zero latency: Real-time synchronization of BIM data in virtual reality for collaborative decision-making. Automation in Construction, 85, 51-64. https://doi.org/10.1016/j.autcon.2017.10.009.

Elziny, A. A., Mohamadien, M. A., Ibrahim, H. M., \& Abdel Fattah, M. K. (2016). An expert system to manage dispute resolutions in construction projects in Egypt. Ain Shams Engineering Journal, 7(1), 57-71. https://doi.org/10.1016/j. asej.2015.05.002.

Garcia-Marques, T., Fernandes, A., Fonseca, R., \& Prada, M. (2015). Social presence and the composite face effect. Acta Psychologica, 158, 61-66. https://doi.org/ 10.1016/j.actpsy.2015.04.001

Giles, D. C. (2002). Parasocial interaction: A review of the literature and a model for future research. Media Psychology, 4(3), 279-305. https://doi.org/10.1207/ S1532785XMEP0403_04.

Grimm, V., \& Mengel, F. (2011). Let me sleep on it: Delay reduces rejection rates in ultimatum games. Economics Letters, 111(2), 113-115. https://doi.org/10. 1016/j.econlet.2011.01.025.

Gunawardena, C. N. (1995). Social presence theory and implications for interaction and collaborative learning in computer conferences. International Journal of Educational Telecommunications, 1(2/3), 147-166.

Gunawardena, C. N., \& Zittle, F. J. (1997). Social presence as a predictor of satisfaction within a computer-mediated conferencing environment. American Journal of Distance Education, 11(3), 8-26. https://doi.org/10.1080/ 08923649709526970.

Heeter C (1992). Being there: The subjective experience of presence. Presence: Teleoperators and Virtual Environments, 1(2), 262-271.

Heeter, C. (1995). Communication research on consumer VR. In LEA's communication series. Communication in the age of virtual realit (pp. 191-218).

Husserl, E. (1973). Cartesian mediations: An introduction to phenomenology. The Hauge: Martinus Nijhoff

Jaffar, N. Tharim, A. H. A., \& Shuib, M. N. (2011). Factors of conflict in construction industry: A literature review. Procedia Engineering, 20, 193-202. https://doi. org/10.1016/j.proeng.2011.11.156.

Jarvenpaa, S. L., Knoll, K., \& Leidner, D. E. (1998). Is anybody out there? Antecedents of Trust in Global Virtual Teams. Journal of Management Information Systems, 14(4), 29-64. https://doi.org/10.1080/07421222.1998. 11518185.

Joseph, S., \& Janet, F. (1991). Organizational colleagues, media richness, and electronic mail: A test of the social influence model of technology use. Communication Research, 18(4), 487-523. https://doi.org/10.1177/ 009365091018004003

Kishi, M. (2008). Perceptions and use of electronic media: Testing the relationship between organizational interpretation differences and media richness. Information \& Management, 45(5), 281-287. https://doi.org/10.1016/j.im.2008.02.008.

Klimmt, C., Hartmann, T., \& Schramm, H. (2006). Parasocial interactions and relationships.

Lee, K., \& Nass, C. (2003). Designing social presence of social actors in human computer interaction, Computer Human Interaction 2003 (Vol. 5).

Lester, E. I. A. (2017). Chapter 42 - conflict management and dispute resolution. In Project Management, planning and control (7th ed., pp. 393-399). Oxford: Butterworth-Heinemann.

Liberman, N., \& Trope, Y. (2014). Traversing psychological distance. Trends in Cognitive Sciences, 18(7), 364-369. https://doi.org/10.1016/j.tics.2014.03.001.

Liberman, N., Trope, Y., \& Stephan, E. (2007). Chapter 15: Psychological Distance.

Liu, S.-H., Liao, H.-L., \& Pratt, J. A. (2009). Impact of media richness and flow on elearning technology acceptance. Computers \& Education, 52(3), 599-607. https://doi.org/10.1016/j.compedu.2008.11.002.

Lombard, M., \& Ditton, T. (1997). At the heart of it all: The concept of presence. Journal of Computer-Mediated Communication, 3(2). https://doi.org/10.1111/j. 1083-6101.1997.tb00072.x. 
Loomis, J. M., Blascovich, J. J., \& Beall, A. C. (1999). Immersive virtual environment technology as a basic research tool in psychology. Behavior Research Methods, Instruments, \& Computers, 31(4), 557-564. https://doi.org/10.3758/ BF03200735.

Lowenthal, P. L. (2009). The evolution and influence of social presence theory on online Learning. University of Colorado Denver: CU Online. Hershey, PA: IGI Global

Lu, W., Zhang, L., \& Pan, J. (2015). Identification and analyses of hidden transaction costs in project dispute resolutions. International Journal of Project Management, 33(3), 711-718. https://doi.org/10.1016/j.jproman.2014.08.009.

Lu, Y., Kim, Y., Dou, X., \& Kumar, S. (2014). Promote physical activity among college students: Using media richness and interactivity in web design. Computers in Human Behavior, 41, 40-50. https://doi.org/10.1016/j.chb.2014.08.012.

Mayer, R. C., Davis, J. H., \& Schoorman, F. D. (1995). An integrative model of organizational trust. The Academy of Management Review, 20(3), 709-734. https://doi.org/10.2307/258792.

Mele, C. (2011). Conflicts and value co-creation in project networks. Industrial Marketing Management, 40(8), 1377-1385. https://doi.org/10.1016/j. indmarman.2011.06.033.

Mesa, H. A., Molenaar, K. R., \& Alarcón, L. F. (2016). Exploring performance of the integrated project delivery process on complex building projects. International Journal of Project Management, 34(7), 1089-1101. https://doi.org/ 10.1016/j.ijproman.2016.05.007.

Mitkus, S., \& Mitkus, T. (2014). Causes of conflicts in a construction industry: A communicational approach. Procedia - Social and Behavioral Sciences, 110 777-786. https://doi.org/10.1016/j.sbspro.2013.12.922.

Mossalam, A. (2017). Projects' issue management. HBRC Journal. https://doi.org/ 10.1016/j.hbrcj.2017.12.001.

Nass, C., \& Moon, Y. (2000). Machines and mindlessness: Social responses to computers. Journal of Social Issues, 56(1), 81-103.

Niazi, G. A., \& Painting, N. (2017). Significant factors causing cost overruns in the construction industry in Afghanistan. Procedia Engineering, 182, 510-517. https://doi.org/10.1016/j.proeng.2017.03.145.

Norouzi, N., Shabak, M., Embi, M. R. B., \& Khan, T. H. (2015). The architect, the client and effective communication in architectural design practice. Procedia - Social and Behavioral Sciences, 172, 635-642. https://doi.org/10.1016/j. sbspro.2015.01.413

Nowak, K. L., \& Biocca, F. (2003). The effect of the agency and anthropomorphism on Users' sense of telepresence, Copresence, and social presence in virtual environments. Presence: Teleoperators and Virtual Environments, 12(5), 481494. https://doi.org/10.1162/105474603322761289.

Odeh, A. M., \& Battaineh, H. T. (2002). Causes of construction delay: Traditional contracts. International Journal of Project Management, 20(1), 67-73. https:// doi.org/10.1016/S0263-7863(00)00037-5.

Otondo, R. F., Van Scotter, J. R., Allen, D. G., \& Palvia, P. (2008). The complexity of richness: Media, message, and communication outcomes. Information \& Management, 45(1), 21-30. https://doi.org/10.1016/j.im.2007.09.003.

Palme, J. (2011). Before the Internet: Early Experiences of Computer Mediated Communication. In Paper presented at the History of Nordic Computing 3, Berlin, Heidelberg.

Palvia, P., Pinjani, P., Cannoy, S., \& Jacks, T. (2011). Contextual constraints in media choice: Beyond information richness. Decision Support Systems, 51(3), $657-$ 670. https://doi.org/10.1016/j.dss.2011.03.006.

Rafaeli, S. (1988). Interactivity: From media to communication. Sage Annual Review of Communicatoin Research: Advancing Communication Science, 16, 110-134.

Rauzana, A. (2016). Causes of conflicts and disputes in construction projects. Journal of Mechanical and Civil Engineering, 13(5), 44-48.

Reyt, J.-N., Wiesenfeld, B. M., \& Trope, Y. (2016). Big picture is better: The social implications of construal level for advice taking. Organizational Behavior and Human Decision Processes, 135, 22-31. https://doi.org/10.1016/j.obhdp.2016.05.004.

Roberts, T. L., Lowry, P. B., \& Sweeney, P. D. (2006). An evaluation of the impact of social presence through group size and the use of collaborative software on group member "voice" in face-to-face and computer-mediated task groups. IEEE Transactions on Professional Communication, 49(1), 28-43. https://doi.org/ 10.1109/TPC.2006.870460

Rubin, R., \& McHugh, M. P. (1987). Development of Parasocial Interaction Relationships (Vol. 31)

Saat, R. M., \& Selamat, M. H. (2014). An examination of Consumer's attitude towards corporate social responsibility (CSR) web communication using media richness theory. Procedia - Social and Behavioral Sciences, 155, 392-397. https://doi.org/10.1016/j.sbspro.2014.10.311
Santos Torres, A., Zarraonandia, T., Díaz, P., \& Aedo, I. (2017). A Comparative Study of Menus in Virtual Reality Environments.

Sarhadi, M. (2016). Comparing communication style within project teams of three project-oriented organizations in Iran. Procedia - Social and Behavioral Sciences, 226, 226-235. https://doi.org/10.1016/j.sbspro.2016.06.183.

Schroeder, R. (2002). Social interaction in virtual environments: Key issues, common themes, and a framework for research. In R. Schroeder (Ed.), The Social Life of Avatars. Computer Supported Cooperative Wor. https://doi.org/ 10.1007/978-1-4471-0277-9 1.

Short, J., Williams, E., \& Christie, B. (1976). The social psychology of telecommunications. London: Wiley.

Steuer, J. (1992). Defining virtual reality: Dimensions determining telepresence. Journal of Communication, 4, 73-93.

Steven, S. L., \& Hang-Yue, N. (2004). The role of trust and contractual safeguards on cooperation in non-equity alliances. Journal of Management, 30(4), 471 485. https://doi.org/10.1016/j.jm.2004.02.002.

Suh, K. S. (1999). Impact of communication medium on task performance and satisfaction: An examination of media-richness theory. Information \& Management, 35(5), 295-312. https://doi.org/10.1016/S0378-7206(98)00097-4.

Sun, P.-C., \& Cheng, H. K. (2007). The design of instructional multimedia in elearning: A media richness theory-based approach. Computers \& Education, 49(3), 662-676. https://doi.org/10.1016/j.compedu.2005.11.016.

Suprapto, M., Bakker, H. L. M., Mooi, H. G., \& Moree, W. (2015). Sorting out the essence of owner-contractor collaboration in capital project delivery. International Journal of Project Management, 33(3), 664-683. https://doi.org/ 10.1016/j.jiproman.2014.05.001.

Sutcliffe, A. G., Binder, J. F., \& Dunbar, R. I. M. (2018). Activity in social media and intimacy in social relationships. Computers in Human Behavior, 85, 227-235. https://doi.org/10.1016/j.chb.2018.03.050.

Tabassi, A. A., Bryde, D. J., Abdullah, A., \& Argyropoulou, M. (2017). Conflict management style of team leaders in multi-cultural work environment in the construction industry. Procedia Computer Science, 121, 41-46. https://doi.org/ 10.1016/j.procs.2017.11.007.

Trope, Y., \& Liberman, N. (2010). Construal-level theory of psychological distance. Pscyhological Review, 117(2), 440463.

Vaaland, T. I. (2004). Improving project collaboration: Start with the conflicts. International Journal of Project Management, 22(6), 447-454. https://doi.org/ 10.1016/j.ijproman.2003.11.003.

Van Os, A., Van Berkel, F., De Gilder, D., Van Dyck, C., \& Groenewegen, P. (2015). Project risk as identity threat: Explaining the development and consequences of risk discourse in an infrastructure project. International Journal of Project Management, 33(4), 877-888. https://doi.org/10.1016/j.jproman.2014.10.016.

Vendemia, M. A. (2017). When do consumers buy the company? Perceptions of interactivity in company-consumer interactions on social networking sites. Computers in Human Behavior, 71, 99-109. https://doi.org/10.1016/j.chb.2017. 01.046.

Verčič, D., Tench, R., \& Tkalac Verčič, A. (2017). Collaboration and conflict between agencies and clients. Public Relations Review. https://doi.org/10.1016/j.pubrev. 2017.11.001.

Wang, W. (2017). Smartphones as social actors? Social dispositional factors in assessing anthropomorphism. Computers in Human Behavior, 68, 334-344. https://doi.org/10.1016/j.chb.2016.11.022.

Weiner, M., \& Mehrabian, A. (1968). Language within language: Immediacy, a channel in verabl communication. New York: Appleton.

Weinhofer, M. (2007). The role of Trust in Strategic Alliances.

Wu, G., Liu, C., Zhao, X., \& Zuo, J. (2017). Investigating the relationship between communication-conflict interaction and project success among construction project teams. International Journal of Project Management, 35(8), 1466-1482. https://doi.org/10.1016/j.ijproman.2017.08.006.

Xu, Q., \& Sundar, S. S. (2016). Interactivity and memory: Information processing of interactive versus non-interactive content. Computers in Human Behavior, 63 , 620-629. https://doi.org/10.1016/j.chb.2016.05.046.

Zulch, B. G. (2014). Communication: The Foundation of project management. Procedia Technology, 16, 1000-1009. https://doi.org/10.1016/j.protcy.2014.10 054. 